This item was submitted to Loughborough's Institutional Repository (https://dspace.lboro.ac.uk/) by the author and is made available under the following Creative Commons Licence conditions.

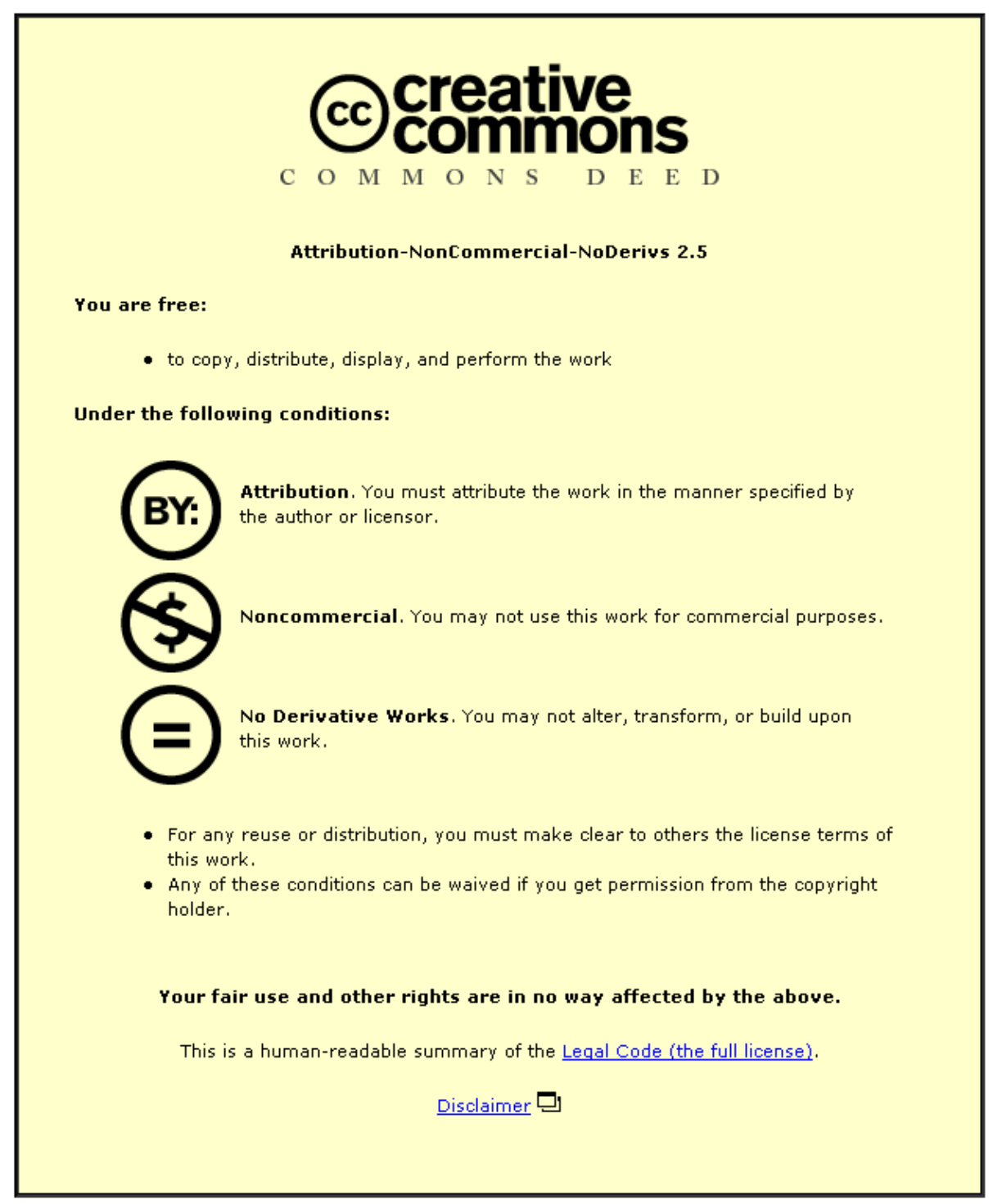

For the full text of this licence, please go to: http://creativecommons.org/licenses/by-nc-nd/2.5/ 


\title{
A New Variable Tap-Length LMS Algorithm to Model an Exponential Decay Impulse Response
}

\author{
Yonggang Zhang, Student Member, IEEE, Jonathon A. Chambers, Senior Member, IEEE, \\ Saeid Sanei, Senior Member, IEEE, Paul Kendrick, and Trevor J. Cox
}

\begin{abstract}
This letter proposes a new variable tap-length leastmean-square (LMS) algorithm for applications in which the unknown filter impulse response sequence has an exponential decay envelope. The algorithm is designed to minimize the mean-square deviation (MSD) between the optimal and adaptive filter weight vectors at each iteration. Simulation results show the proposed algorithm has a faster convergence rate as compared with the fixed tap-length LMS algorithm and is robust to the initial tap-length choice.
\end{abstract}

Index Terms-Adaptive filters, variable tap-length least-meansquare (LMS) algorithm.

\section{INTRODUCTION}

$\mathbf{T}$ HE least-mean-square (LMS) algorithm has been extensively used in many applications as a consequence of its simplicity and robustness [1], [2]. In many applications of the LMS algorithm, the tap-length of the adaptive filter is kept fixed. However, in certain applications, the tap-length of the optimal filter is unknown or even variable. According to the analysis in [3] and [4], the mean-square error (MSE) is likely to increase if the tap-length is undermodeled. To avoid such a situation, a sufficiently large filter tap-length is needed. However, the computational cost is proportional to the tap-length; thus, a variable tap-length algorithm is needed to find a proper choice of the tap-length.

In many applications such as echo cancelling, the unknown filter exhibits a constant exponential decay envelope. Modeling the unknown impulse response in such applications is typically achieved with a length $N$ finite impulse response (FIR) filter, denoted by $\mathbf{c}_{N}$. In practice, $N$ is chosen as a compromise between modeling the significant energy within the impulse response and limiting computational complexity. In this letter, we seek an adaptive solution for the choice of $N$. The evolution of the tap-length of our algorithm is designed in an adaptive way to minimize the mean-square deviation (MSD) at each iteration, which is defined as $E\left\{\left\|\mathbf{c}_{N}-\mathbf{w}_{N}(n)\right\|_{2}^{2}\right\}$, where $\mathbf{w}_{N}(n)$ is the adaptive filter weight vector, $\|\cdot\|_{2}^{2}$ denotes the squared Euclidean

Manuscript received June 26, 2006; revised September 9, 2006. This work was supported by the Engineering and Physical Sciences Research Council (EPSRC) under Grant GR/S77547/01. The associate editor coordinating the review of this manuscript and approving it for publication was Prof. Benoit Champagne.

Y. Zhang, J. A. Chambers, and S. Sanei are with the Center of Digital Signal Processing, Cardiff School of Engineering, Cardiff University, Wales CF24 3AA, U.K. (e-mail: zhangy15@cf.ac.uk; chambersj@cf.ac.uk; saneis@cf.ac.uk).

P. Kendrick and T. J. Cox are with the School of Acoustics and Electronic Engineering, University of Salford, Salford M5 4WT, U.K. (e-mail: P.kendrick@salford.ac.uk; T.J.Cox@salford.ac.uk).

Color versions of Figs. 1-3 are available online at http://ieeexplore.ieee.org.

Digital Object Identifier 10.1109/LSP.2006.887784 norm operator, and $E\{\cdot\}$ represents statistical expectation. The target of our approach is not only to find a good choice of the steady-state tap-length for the adaptive filter but also to ensure well-behaved transient tap-length convergence, so that a better performance as compared with the fixed tap-length algorithm is obtained.

In a previous research study [3], a theoretically optimal variable tap-length sequence for the LMS algorithm in such applications has been introduced. However, this algorithm suffers from heavy computational complexity due to solving for Lambert's W-function [3]; thus, it is not suitable in practice. Other existing variable tap-length algorithms such as [5]-[7] are sensitive to the parameter choice, i.e., different parameters will result in very different performance, according to the discussion in [7]. As will be shown by our simulation results, the proposed algorithm converges faster than the fixed tap-length LMS algorithm and is very robust to the initial tap-length choice.

\section{New Variable Tap-Length LMS Algorithm}

For convenience, we formulate the LMS algorithm with a system identification model and assume that the desired unknown filter impulse response sequence has a constant exponential decay envelope. In this model, the desired signal $d(n)$ is formulated as follows:

$$
d(n)=\mathbf{x}_{N}^{T}(n) \mathbf{c}_{N}+v(n)
$$

where $(\cdot)^{T}$ denotes the transpose operator, $\mathbf{x}_{N}$ is the input vector with a tap length of $N, v(n)$ is the noise signal, and $\mathbf{c}_{N}$ can be modeled as follows:

$$
c(i)=e^{(-(i-1) \tau)} r(i), \quad i=1, \ldots, N
$$

where $c(i)$ is the $i$ th coefficient of the unknown filter vector $\mathbf{c}_{N}$, $\tau$ is a positive constant to model the decay rate, and $r(i)$ is drawn from a zero-mean unit variance Gaussian sequence.

Since in the variable-tap length LMS algorithm, the tap-length is time-varying rather than fixed, we will use $M(n)$ to denote the integer tap-length that is used for the coefficient update of the LMS algorithm at the $n$th iteration and assume $M(n) \leq N$. Note that in [3], the authors used a parameter $M$ to denote the tap-length. By using our notation, the filter coefficients can be updated as

$$
\mathbf{w}_{M(n)}(n+1)=\mathbf{w}_{M(n)}(n)+\mu e(n) \mathbf{x}_{M(n)}(n)
$$

where $\mathbf{w}_{M(n)}$ and $\mathbf{x}_{M(n)}$ are, respectively, the $M(n)$-tap adaptive filter vector and the input vector, $\mu$ is the step size for the update of the coefficients, and $e(n)$ is the output error defined as

$$
e(n)=d(n)-\mathbf{x}_{M(n)}^{T}(n) \mathbf{w}_{M(n)}(n) .
$$


Similar to the formulation in [3], we split $\mathbf{c}_{N}$ into two parts as

$$
\left(\begin{array}{c}
\mathbf{c}_{M(n)}^{\prime} \\
\mathbf{c}_{N-M(n)}^{\prime \prime}
\end{array}\right)
$$

where $\mathbf{c}_{M(n)}^{\prime}$ is the part modeled by $\mathbf{w}_{M(n)}(n)$, and $\mathbf{c}_{N-M(n)}^{\prime \prime}$ is the part undermodeled. If we define $\mathbf{g}_{N}(n)$ as the total coefficient error vector $\mathbf{c}_{N}-\mathbf{w}_{N}(n)$, where $\mathbf{w}_{N}(n)$ is obtained by padding $\mathbf{w}_{M(n)}(n)$ with zeros, the MSD can then be formulated by $E\left\{\left\|\mathbf{g}_{N}(n)\right\|_{2}^{2}\right\}$.

Since the tap-length should not be constrained to be an integer to find a continuous update, similar to as in [3] and [7], we use the fractional-tap length concept in the following derivations, where the fractional-tap length denoted by $M_{f}(n)$ will be used in the update of the tap-length, and the tap-length $M(n)$ that is used for the update of the adaptive filter coefficients is assigned to the integer immediately below $M_{f}(n)$.

Similar to [3], we assume that both the input signal $x(n)$ and the noise signal $v(n)$ are statistically independent identically distributed (i.i.d.) zero-mean Gaussian white noise signals with variances $\sigma_{x}^{2}$ and $\sigma_{v}^{2}$, respectively. According to the analysis in [3], we know that the evolution of the MSD can be formulated as follows:

$$
\begin{aligned}
E\left\{\left\|\mathbf{g}_{N}(n+1)\right\|_{2}^{2}\right\}=\beta & E\left\{\left\|\mathbf{g}_{N}(n)\right\|_{2}^{2}\right\} \\
& +(\eta-\beta)\left\|\mathbf{c}_{N-M_{f}(n+1)}^{\prime \prime}\right\|_{2}^{2}+\gamma
\end{aligned}
$$

where

$$
\begin{aligned}
& \beta=1-2 \mu \sigma_{x}^{2}+\left(M_{f}(n+1)+2\right) \mu^{2} \sigma_{x}^{4} \\
& \eta=1+M_{f}(n+1) \mu^{2} \sigma_{x}^{4}
\end{aligned}
$$

and

$$
\gamma=M_{f}(n+1) \mu^{2} \sigma_{x}^{2} \sigma_{v}^{2}
$$

The range of the step size that ensures the convergence of (5) is [3]

$$
0<\mu<\frac{2}{\left(M_{f}(n+1)+2\right) \sigma_{x}^{2}} .
$$

A theoretical optimal tap-length sequence for the LMS algorithm has been given in [3] by minimizing the MSD at each iteration, according to (5). Based on this work, next, we will derive a new practical optimal variable tap-length LMS algorithm in an adaptive form. The motivation of this approach is that with a given current tap length, we may find the tap-length for the next iteration, so the MSD will be minimized.

\section{A. New Variable Tap Length Algorithm}

At first, to speed up the convergence rate of the LMS algorithm, the step size is made variable rather than fixed, according to the range of $\mu$ described in (9). In [3], the step size is set to $\mu(n)=\mu^{\prime} /\left(\left(M_{f}(n)+2\right) \sigma_{x}^{2}\right)$, where $\mu^{\prime}$ is a fixed constant and less than two. To remove the dependence between the step size $\mu(n)$ and the tap length $M_{f}(n)$, and noting that $M_{f}(n-1)$ is very close to $M(n)$, we set the step size as follows:

$$
\mu(n)=\frac{\mu^{\prime}}{\left(\left(M_{f}(n-1)+\delta\right) \sigma_{x}^{2}\right)}
$$

where $\delta$ is an integer larger than two to ensure stability of the algorithm.
Second, the squared norm of the partial response of $\mathbf{c}_{N}$ can be expressed in the form [3]

$$
\left\|\mathbf{c}_{N-M_{f}(n)}^{\prime \prime}\right\|_{2}^{2}=\frac{e^{-2 M_{f}(n) \tau}-e^{-2 N \tau}}{1-e^{-2 N \tau}}\left\|\mathbf{c}_{N}\right\|_{2}^{2} .
$$

As shown in [3], the second-order derivative $\left(\partial E\left\{\left\|\mathbf{g}_{N}(n+1)\right\|_{2}^{2}\right\} / \partial M_{f}^{2}(n+1)\right)$ is positive; thus, a tap-length $M_{f}(n+1)$ is existing to minimize the term $E\left\{\left\|\mathbf{g}_{N}(n+1)\right\|_{2}^{2}\right\}$. Replacing $\mu$ in (5) with $\mu(n+1)$, substituting (6), (7), (8), (11) into (5), and setting $\left(\partial E\left\{\left\|\mathbf{g}_{N}(n+1)\right\|_{2}^{2}\right\} / \partial M_{f}(n+1)\right)=0$, we can obtain $\mu(n+1) \sigma_{x}^{2} E\left\{\left\|\mathbf{g}_{N}(n)\right\|_{2}^{2}\right\}-4 \tau\left(1-\mu(n+1) \sigma_{x}^{2}\right)$

$$
\times \frac{e^{-2 M_{f}(n+1) \tau}}{1-e^{-2 N \tau}}\left\|\mathbf{c}_{N}\right\|_{2}^{2}+M_{f}(n+1) \mu(n+1) \sigma_{v}^{2}=0 .
$$

After rearranging, we can find

$$
\begin{aligned}
& M_{f}(n+1)=-\frac{1}{2 \tau} \\
& \quad \times \log \frac{\mu(n+1) \sigma_{x}^{2} E\left\{\left\|\mathbf{g}_{N}(n)\right\|_{2}^{2}\right\}+\mu(n+1) \sigma_{v}^{2}}{4 \tau\left(1-\mu(n+1) \sigma_{x}^{2}\right) \frac{\left\|\mathbf{c}_{N}\right\|_{2}^{2}}{1-e^{-2 N \tau}}} .
\end{aligned}
$$

Substituting (10) into (13), we have

$$
M_{f}(n+1)=-\frac{1}{2 \tau} \log \frac{\mu^{\prime} \sigma_{x}^{2} E\left\{\left\|\mathbf{g}_{N}(n)\right\|_{2}^{2}\right\}+\mu^{\prime} \sigma_{v}^{2}}{4 \tau \sigma_{x}^{2}\left(M_{f}(n)+\delta-\mu^{\prime}\right) \frac{\left\|\mathbf{c}_{N}\right\|_{2}^{2}}{1-e^{-2 N \tau}}} .
$$

If we define $\Delta M_{f}=M_{f}(n+1)-M_{f}(n)$, from (14), we obtain that

$$
\begin{aligned}
& \Delta M_{f}=-\frac{1}{2 \tau} \\
& \times \log \frac{\left(\sigma_{x}^{2} E\left\{\left\|\mathbf{g}_{N}(n)\right\|_{2}^{2}\right\}+\sigma_{v}^{2}\right)\left(M_{f}(n-1)+\delta-\mu^{\prime}\right)}{\left(\sigma_{x}^{2} E\left\{\left\|\mathbf{g}_{N}(n-1)\right\|_{2}^{2}\right\}+\sigma_{v}^{2}\right)\left(M_{f}(n)+\delta-\mu^{\prime}\right)} .
\end{aligned}
$$

Thus, we obtain the update of the tap-length of the new variable tap-length LMS algorithm

$$
\begin{aligned}
& M_{f}(n+1)=M_{f}(n)-\frac{1}{2 \tau} \\
& \times \log \frac{\left(\sigma_{x}^{2} E\left\{\left\|\mathbf{g}_{N}(n)\right\|_{2}^{2}\right\}+\sigma_{v}^{2}\right)\left(M_{f}(n-1)+\delta-\mu^{\prime}\right)}{\left(\sigma_{x}^{2} E\left\{\left\|\mathbf{g}_{N}(n-1)\right\|_{2}^{2}\right\}+\sigma_{v}^{2}\right)\left(M_{f}(n)+\delta-\mu^{\prime}\right)} .
\end{aligned}
$$

If we assume that the input signals are independent of the adaptive weight coefficients, we can show that [1]

$$
E\left\{e^{2}(n)\right\}=\sigma_{x}^{2} E\left\{\left\|\mathbf{g}_{N}(n)\right\|_{2}^{2}\right\}+\sigma_{v}^{2} .
$$

Substituting (17) into (16), we have

$$
\begin{aligned}
M_{f}(n+1)= & M_{f}(n)-\frac{1}{2 \tau} \\
& \times \log \frac{E\left\{e^{2}(n)\right\}\left(M_{f}(n-1)+\delta-\mu^{\prime}\right)}{E\left\{e^{2}(n-1)\right\}\left(M_{f}(n)+\delta-\mu^{\prime}\right)} .
\end{aligned}
$$

In practice, the statistical average term $E\left\{e^{2}(n)\right\}$ can be approximated by its time average estimation $\overline{e^{2}(n)}$, which can be obtained as

$$
\overline{e^{2}(n)}=\phi \overline{e^{2}(n-1)}+(1-\phi) e^{2}(n)
$$


where $\phi$ is a positive constant close to but less than unity. The new variable-tap length algorithm is then obtained as follows:

$$
M_{f}(n+1)=M_{f}(n)-\frac{1}{2 \tau} \log \frac{\overline{e^{2}(n)}\left(M_{f}(n-1)+\delta-\mu^{\prime}\right)}{\overline{e^{2}(n-1)}\left(M_{f}(n)+\delta-\mu^{\prime}\right)} .
$$

Since the tap length that is used in the update of the filter coefficients must be an integer, we choose the floor of $M_{f}(n+1)$ for the coefficient update

$$
M(n+1)=\left\lfloor M_{f}(n+1)\right\rfloor
$$

where $\lfloor$.$\rfloor is the floor operator that rounds down the embraced$ value to the nearest integer. If we replace the $\mu$ in (3) with $\mu(n)$, the full adaptive algorithm can consequently then be implemented by (3), (4), (10), (19), (20), and (21).

\section{B. Steady-State Performance of the Proposed Algorithm}

According to the update (20), it is straightforward to obtain that

$$
M_{f}(\infty)=M_{f}(0)-\frac{1}{2 \tau} \log \frac{\overline{e^{2}(\infty)}\left(M_{f}(0)+\delta-\mu^{\prime}\right)}{\overline{e^{2}(0)}\left(M_{f}(\infty)+\delta-\mu^{\prime}\right)}
$$

where $M_{f}(0)$ and $M_{f}(\infty)$ are the initial and steady-state values of the fractional tap-length, and $\overline{e^{2}(0)}$ and $\overline{e^{2}(\infty)}$ are the initial and steady-state values of the smoothed square error. The initial value $\overline{e^{2}(0)}$ can be set as $\sigma_{d}^{2}+\sigma_{v}^{2}$, where $\sigma_{d}^{2}$ is the variance of the desired signal and can be formulated by $\sigma_{x}^{2}\left\|\mathbf{c}_{N}\right\|_{2}^{2}$. Substituting (17) into (22), we have

$$
\begin{aligned}
& M_{f}(\infty)=M_{f}(0)-\frac{1}{2 \tau} \\
& \quad \times \log \frac{\left(\sigma_{x}^{2} E\left\{\left\|\mathbf{g}_{N}(\infty)\right\|_{2}^{2}\right\}+\sigma_{v}^{2}\right)\left(M_{f}(0)+\delta-\mu^{\prime}\right)}{\left(\sigma_{x}^{2}\left\|\mathbf{c}_{N}\right\|_{2}^{2}+\sigma_{v}^{2}\right)\left(M_{f}(\infty)+\delta-\mu^{\prime}\right)} .
\end{aligned}
$$

From (5)-(8) and (10), we can have (24), shown at the bottom of the page.

To simplify the formulation, we assume $N$ is very large and $M_{f}(\infty)$ is close to $N$; thus, $M_{f}(\infty) \approx M_{f}(\infty)+2 \approx$ $M_{f}(\infty)+\delta-\mu^{\prime}$ and the term $\left\|\mathbf{c}_{N-M_{f}(\infty)}^{\prime \prime}\right\|_{2}^{2}$ is very small. Furthermore, if we assume that $\mu^{\prime}$ has been chosen properly so that the term $2 \sigma_{x}^{2}\left\|\mathbf{c}_{N-M_{f}(\infty)}^{\prime \prime}\right\|_{2}^{2} \ll \mu^{\prime} \sigma_{v}^{2}$, we obtain

$$
E\left\{\left\|\mathrm{~g}_{N}(\infty)\right\|_{2}^{2}\right\} \approx \frac{\mu^{\prime} \sigma_{v}^{2}}{\left(2-\mu^{\prime}\right) \sigma_{x}^{2}} .
$$

Substituting (25) into (23), we have

$$
\begin{aligned}
& M_{f}(\infty) \approx M_{f}(0)-\frac{1}{2 \tau} \\
& \quad \times \log \frac{2 \sigma_{v}^{2}\left(M_{f}(0)+\delta-\mu^{\prime}\right)}{\left(\sigma_{x}^{2}\left\|\mathbf{c}_{N}\right\|_{2}^{2}+\sigma_{v}^{2}\right)\left(M_{f}(\infty)+\delta-\mu^{\prime}\right)\left(2-\mu^{\prime}\right)} .
\end{aligned}
$$

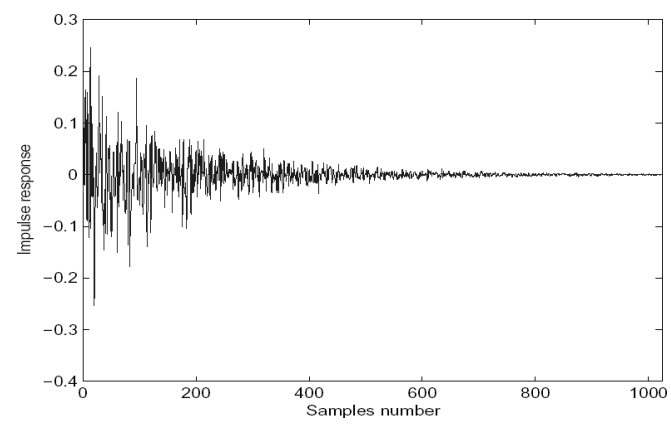

Fig. 1. One representation of the unknown impulse response sequence.

From (26), we know that the steady-state tap-length $M_{f}(\infty)$ is correlated with three parameters: the step size $\mu^{\prime}$, the parameter $\delta$, and the initial tap-length $M_{f}(0)$. If we assume that $\mu^{\prime}$ has been chosen properly, and both $M(\infty)$ and $M(0)$ are much larger than $\mu^{\prime}$ and $\delta$, then the influence of $\delta$ and $\mu^{\prime}$ can be ignored. Next we will show by simulations that with wide range in the choice of the initial tap-length $M(0)$, the steady-state tap-length $M(\infty)$ can converge to values that provide a good compromise between modeling the significant energy within the impulse response and limiting computational complexity.

\section{SimULATION}

In this section, we will examine the above derivations and compare the proposed algorithm with the fixed tap-length LMS algorithm and the optimal variable tap-length LMS algorithm [3] by simulations. The setup of all the simulations is similar to that in [3]: the unknown filter is a white Gaussian noise sequence with zero mean and a variance of 0.01 weighted by an exponential decay envelope. The tap-length is set to 1024 , and the decay parameter $\tau$ is set to 0.005 . One representation of the unknown filter can be seen in Fig. 1. The input signal is another white Gaussian noise sequence with zero mean and unit variance. The noise signal is a zero-mean random Gaussian sequence with a variance of 0.01 . The parameter $\delta$ for the proposed algorithm is set to 5 . The smoothing parameter $\phi$ in (19) is set to 0.99 . The step size $\mu^{\prime}$ for both the proposed algorithm and the optimal variable tap-length algorithm is set to 0.5 . The initial value of $\overline{e^{2}(n)}$, i.e., $e^{2}(0)$, is set to $\sigma_{d}^{2}+\sigma_{v}^{2}$.

The steady-state MSD with different steady-state tap-length values is shown in Fig. 2(a), calculated from (24). It is clear to see in Fig. 2(a) that the steady-state MSD decreases with the increase of the steady state tap-length. However, due to the exponential damping envelope structure as shown in Fig. 1, the MSD will nearly be a constant if the steady-state tap-length is larger than some value, such as 800 in our simulation. The main energy of the unknown impulse response is contained in approximately the first 800 coefficients, which is the part we want to find by our approach.

The values of the steady-state tap-length with different initial tap-length values are shown in Fig. 2(b), calculated from (26).

$$
E\left\{\left\|\mathrm{~g}_{N}(\infty)\right\|_{2}^{2}\right\}=\frac{2 \sigma_{x}^{2}\left(M_{f}(\infty)+2-\mu^{\prime}\right)\left\|\mathbf{c}_{N-M_{f}(\infty)}^{\prime \prime}\right\|_{2}^{2}+\mu^{\prime} M_{f}(\infty) \sigma_{v}^{2}}{\left(2-\mu^{\prime}\right)\left(M_{f}(\infty)+2\right) \sigma_{x}^{2}}
$$




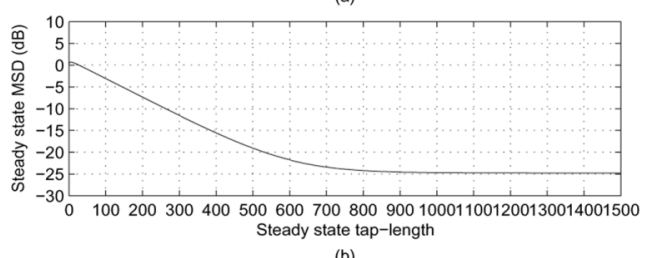

(b)

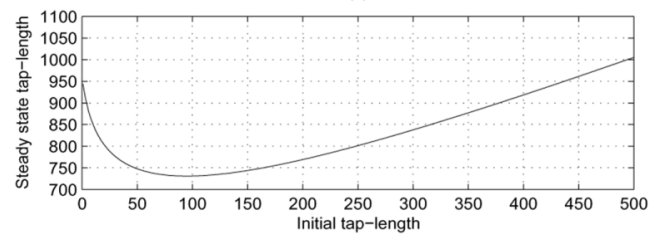

Fig. 2. (a) Steady-state MSD with different values of the steady-state tap-length according to (24). (b) Steady-state tap-lengths with different initial tap-length values according to (26).

We can see from Fig. 2(b), together with Fig. 2(a), that with a wide range of the initial tap-length, the steady-state tap-length can converge to some values that give good compromise between modeling the significant energy within the impulse response and limiting computational complexity; thus, we conclude that the proposed algorithm is robust to the choice of the initial tap-length.

To confirm (26), we perform several simulations for the proposed algorithm with different initial tap-length values. The evolution curves of the tap-length with different initial tap-length values are shown in Fig. 3(a), where different initial tap-length values, simulated steady-state tap-length values, and the theoretical steady-state tap-length values that are obtained from (26) are given in the legend of the plot. As a comparison, the optimal variable tap-length is also given. From these values, we can see that the simulated steady-state tap-lengths match the theoretical values quite well. Furthermore, the initial parts of all the variable tap-length evolution curves of the proposed algorithm are similar to that of the optimal variable tap-length sequence.

Finally, the proposed algorithm is compared with the fixed tap-length LMS algorithm and the optimal variable tap-length algorithm by comparing the MSD. Two experiments are performed for the fixed tap-length algorithm with different taplengths. In one experiment, the tap-length is set to 1024 , and the step size is set to $0.5 / 1024$. In another experiment, the tap-length is set to 512, and the step size is set to $0.5 / 512$. The initial tap-length of the proposed algorithm is set to 20 . The evolution curves of the MSD of the fixed tap-length LMS algorithm, the optimal variable tap-length LMS algorithm, and the proposed algorithm are shown in Fig. 3(b). All the results in Fig. 3 are obtained by averaging the results over 100 Monte Carlo trials of the same experiment.

From Fig. 3(b), we can find that although the steady-state taplength of the proposed algorithm is less than that of the optimal variable tap-length algorithm, their MSD evolution curves are nearly the same. It is clear to see that with a similar steadystate MSD, the proposed algorithm converges faster than the fixed tap-length LMS algorithm with a tap-length of 1024. The convergence rate of the fixed tap-length LMS algorithm with a tap-length of 512 is fast, but the MSD is large. The proposed algorithm has both fast convergence rate and small MSD, and a good steady-state tap-length is also found in an adaptive way.

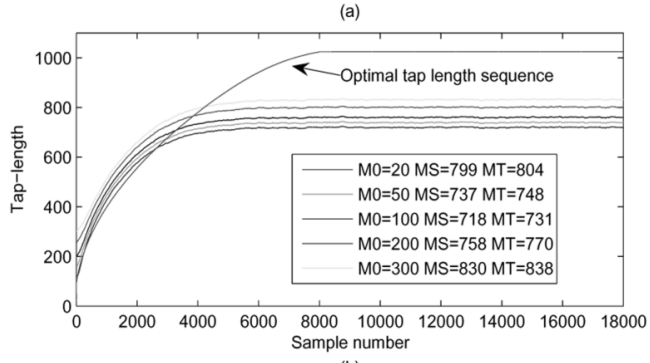

(b)

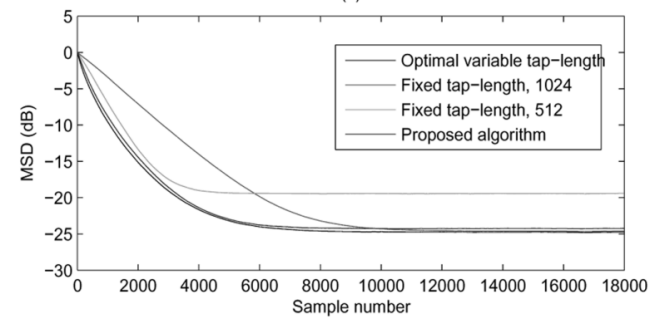

Fig. 3. (a) Optimal variable tap-length sequence obtained from [3] and the evolution curves of the tap-length of the proposed method with different initial tap-lengths (M0: initial tap length; MS: simulated steady-state result; MT: theoretical steady-state result). (b) Evolution curves of the MSD of the fixed tap-length LMS algorithm, optimal variable tap-length LMS algorithm, and the proposed algorithm.

\section{CONCLUSION}

A new variable tap-length LMS algorithm has been introduced in this letter. This algorithm is based on the assumption of an exponential decay envelope filter impulse response structure and designed to minimize the MSD by using variable tap-length at each iteration. According to the simulation results, the proposed algorithm provides a good approach for the choice of the unknown steady-state tap-length as well as both fast convergence rate and small steady-state MSD.

\section{ACKNOWLEDGMENT}

The authors would like to thank both the reviewers for improving the clarity of the presentation of this letter.

\section{REFERENCES}

[1] B. Farhang-Boroujeny, Adaptive Filters: Theory and Applications. New York: Wiley, 1998.

[2] A. H. Sayed, Fundamentals of Adaptive Filtering. New York: Wiley, 2003.

[3] Y. Gu, K. Tang, H. Cui, and W. Du, "Convergence analysis of a deficient-length LMS filter and optimal-length sequence to model exponential decay impulse response," IEEE Signal Process. Lett., vol. 10, no. 1, pp. 4-7, Jan. 2003.

[4] K. Mayyas, "Performance analysis of the deficient length LMS adaptive algorithm," IEEE Trans. Signal Process., vol. 53, no. 8, pp. 2727-2734, Aug. 2005.

[5] F. Riero-Palou, J. M. Noras, and D. G. M. Cruickshank, "Linear equalisers with dynamic and automatic length selection," Electron. Lett., vol. 37, no. 25, pp. 1553-1554, Dec. 2001.

[6] Y. Gu, K. Tang, H. Cui, and W. Du, "LMS algorithm with gradient descent filter length," IEEE Signal Process. Lett., vol. 11, no. 3, pp. 305-307, Mar. 2004

[7] Y. Gong and C. F. N. Cowan, "An LMS style variable tap-length algorithm for structure adaptation," IEEE Trans. Signal Process., vol. 53, no. 7, pp. 2400-2407, Jul. 2005. 